\title{
Hausdorff Dimension of a Random Attractor for Stochastic Boussinesq Equations with Double Multiplicative White Noises
}

\author{
Yin Li, ${ }^{1,2}$ Ruiying Wei, ${ }^{1,2}$ and Donghong $\mathrm{Cai}^{3}$ \\ ${ }^{1}$ School of Mathematics and Statistics, Shaoguan University, Shaoguan 512005, China \\ ${ }^{2}$ School of Mathematics and Statistics, Yunnan University, Yunnan 650091, China \\ ${ }^{3}$ Department of Mathematics, Southwest Jiaotong University, Chengdu 610031, China
}

Correspondence should be addressed to Ruiying Wei; weiruiying521@163.com

Received 24 January 2016; Revised 27 April 2016; Accepted 28 April 2016

Academic Editor: Maria Alessandra Ragusa

Copyright (C) 2016 Yin Li et al. This is an open access article distributed under the Creative Commons Attribution License, which permits unrestricted use, distribution, and reproduction in any medium, provided the original work is properly cited.

This paper investigates the existence of random attractor for stochastic Boussinesq equations driven by multiplicative white noises in both the velocity and temperature equations and estimates the Hausdorff dimension of the random attractor.

\section{Introduction}

In this paper, we consider the following $2 \mathrm{D}$ stochastic Boussinesq equation on $D=(0,1) \times(0,1) \subset R^{2}$ :

$$
\begin{aligned}
& \begin{array}{l}
d v+ \\
+
\end{array} \quad T e_{2} d t+b_{1} v \circ d W_{1}(t), \\
& \begin{aligned}
d T & +[(v \cdot \nabla) T-k \Delta T] d t=b_{2} T \circ d W_{2}(t),
\end{aligned} \\
& \operatorname{div} v=0, \\
& \left.v\right|_{t=t_{0}}=v_{0}, \\
& \left.T\right|_{t=t_{0}}=T_{0},
\end{aligned}
$$

where $v=\left(v_{1}(x, t), v_{2}(x, t)\right)$ denotes the velocity of the fluid; $T(x, t)$ is the temperature; and function $p(x, t)$ represents the pressure. We set $(x, t) \in D \times R$, and $\left\{e_{1}, e_{2}\right\}$ is the canonical basis of $R^{2}$. Let us consider $\mu, k>0$ the Grashof and Prandtl numbers. $W(t)=\left(W_{1}(t), W_{2}(t)\right)^{T}$ is two-sided real-valued Wiener process on a probability space $\left(\Omega, \mathscr{F},\left\{\mathscr{F}_{t}\right\}_{t \in R}, P\right)$, here

$$
\Omega=\left\{\omega \in C\left(R, R^{2}\right): \omega(0)=0\right\} .
$$

The Borel $\sigma$-algebra $\mathscr{F}$ on $\Omega$ is generated by the compact open topology, and $P$ is the corresponding Wiener measure; $\circ d W_{i}(t)$ denotes the Stratonovich form white noise.
$\mathrm{Pu}$ and Guo in [1] studied the global well-posedness of stochastic $2 \mathrm{D}$ Boussinesq equations with partial viscosity; $\mathrm{Li}$ and Guo [2] consider the stochastic Boussinesq equations, which are influenced by multiplicative white noise in the velocity equations. Recently, Zhao and $\mathrm{Li}$ [3] established the existence of a random attractor for stochastic Boussinesq equations with double additive white noises.

In many cases, the Hausdorff dimension of a random attractor is finite. Crauel and Flandoli [4] developed a technique to estimate the Hausdorff dimension of attractors for certain dynamical systems. But they required the noise to be bounded. Debussche [5] applied a random squeezing property to estimate the Hausdorff dimension without the assumption of bounded noise. A number of authors [6-13] used this method to estimate the Hausdorff dimension of attractors for many stochastic equations.

As far as we know, there are no results on stochastic 2D Boussinesq equations with double multiplicative white noises on bounded domains. In this paper, we prove the existence of random attractor for the corresponding RDS associated with problem (1). And we give an estimate of Hausdorff dimension for the random attractor.

The paper is organized as follows. In the first section, we recall some latest results and what we want to do for the $2 \mathrm{D}$ stochastic Boussinesq equations. We study the RDS determined by (1) in Section 2. In Section 3, we prove the existence of a random attractor. Finally, we estimate the 
Hausdorff dimension of the random attractor in Section 4 .

\section{Random Dynamical System}

The random dynamical system generated by stochastic Boussinesq equations with double multiplicative noise will be studied in this section. Thus, we introduce a process, which enables us to transform the stochastic equations into a deterministic equation with a random parameter.

Let

$$
\begin{aligned}
\alpha_{i}(t) & =e^{-b_{i} W_{i}(t)}, \quad i=1,2, \\
\xi & =\alpha_{1}(t) v \\
\eta & =\alpha_{2}(t) T .
\end{aligned}
$$

From (1), we get the stochastic Boussinesq equations without white noises:

$$
\begin{aligned}
\frac{d \xi}{d t}+\alpha_{1}^{-1}(t)(\xi \cdot \nabla) \xi-\mu \Delta \xi+\alpha_{1}(t) \nabla p & =\frac{\alpha_{1}(t)}{\alpha_{2}(t)} e_{2} \eta, \\
\frac{d \eta}{d t}+\alpha_{1}^{-1}(t)(\xi \cdot \nabla) \eta-k \Delta \eta & =0, \\
\operatorname{div} \xi & =0, \\
\left.\xi\right|_{t=t_{0}} & =\xi_{0}, \\
\left.\eta\right|_{t=t_{0}} & =\eta_{0} .
\end{aligned}
$$

In the present paper, we consider the Hilbert space: $\mathscr{H}=\mathscr{H}_{1} \times$ $\mathscr{H}_{2}$, with the scalar product $(\cdot, \cdot)$ and norm $\|\cdot\|$, where

$$
\begin{aligned}
& \mathscr{H}_{1}=\left\{\xi \in \mathscr{H}_{2}^{2}: \operatorname{div} \xi=0\right\}, \\
& \mathscr{H}_{2}=L^{2}(D) .
\end{aligned}
$$

We also consider the subspace: $V=V_{1} \times V_{2}$. Consider

$$
\begin{aligned}
& V_{1}=\left\{\xi \in V_{2}^{2}: \operatorname{div} \xi=0\right\}, \\
& V_{2}=\text { closure of } H_{0}^{1}(D) \text { in } L^{2}(D)
\end{aligned}
$$

and its scalar product and norms are $((\cdot, \cdot))$ and $\|\cdot\|_{1}$, respectively, for any $\eta_{1}, \eta_{2} \in V_{2}$ :

$$
\begin{aligned}
\left(\left(\eta_{1}, \eta_{2}\right)\right) & =\int_{D} \operatorname{grad} \eta_{1} \operatorname{grad} \eta_{2} d x, \\
\|\eta\|_{1} & =\left((\eta, \eta)^{1 / 2}\right)=\|\nabla \eta\| .
\end{aligned}
$$

Now we give some definitions of common operators. $a$ is the bilinear on $V$ :

$$
\begin{aligned}
& a\left(u_{1}, u_{2}\right)=-\mu\left(\Delta \xi_{1}, \xi_{2}\right)- k\left(\Delta \eta_{1}, \eta_{2}\right), \\
& \forall u_{i}=\left(\xi_{i}, \eta_{i}\right)^{T} \in V, i=1,2 .
\end{aligned}
$$

$A$ is isomorphism from $D(A)$ into $\mathscr{H}$ and from $V$ into the dual space $V^{\prime}$, defined by

$$
\left(A u_{1}, u_{2}\right)=a\left(u_{1}, u_{2}\right), \quad \forall u_{i}=\left(\xi_{i}, \eta_{i}\right)^{T} \in V, i=1,2,
$$

where $D(A)=D\left(A_{1}\right) \times D\left(A_{2}\right)$, being

$$
\begin{aligned}
& D\left(A_{1}\right)=\left\{\xi: \xi \in V_{1} \cap\left(H^{2}(D)\right)^{2}\right\}, \\
& D\left(A_{2}\right)=\left\{\eta: \eta \in V_{2} \cap H^{2}(D)\right\} .
\end{aligned}
$$

We consider trilinear forms $b$ on $V$ defined by

$$
\begin{array}{r}
b\left(u_{1}, u_{2}, u_{3}\right)=-\left(\left(\xi_{1} \cdot \nabla\right) \xi_{2}, \xi_{3}\right)-\left(\left(\xi_{1} \cdot \nabla\right) \eta_{2}, \eta_{3}\right), \\
\forall u_{i}=\left(\xi_{i}, \eta_{i}\right)^{T} \in V, i=1,2 .
\end{array}
$$

$b$ is continuous on $V$ and $\left(\mathscr{H}^{\prime}(D)\right)^{2} \times \mathscr{H}^{\prime}(D)$.

We also define the bilinear continuous operator $B$, which maps $V \times V$ into $V^{\prime}$ and $D(A) \times D(A)$ into $\mathscr{H}$, by

$$
\begin{aligned}
&\left(B\left(u_{1}, u_{2}\right), u_{3}\right)=b\left(u_{1}, u_{2}, u_{3}\right), \\
& \forall u_{i}=\left(\xi_{i}, \eta_{i}\right)^{T} \in V, i=1,2,3 .
\end{aligned}
$$

Finally, we consider a family of linear continuous operators $R(t)=R(t, \omega)$ on $\mathscr{H}$ :

$$
\begin{aligned}
R(t): u & =(\xi, \eta)^{T} \longrightarrow \\
R u & =\left(\frac{\alpha_{1}(t)}{\alpha_{2}(t)} e_{2} \eta, 0\right)^{T} .
\end{aligned}
$$

Assume that $u=(\xi, \eta)^{T}$ is a solution of (4)-(7) and $y=$ $(f, g)^{T}$ is a test function in $V$. Multiplying (4) by $f$ and (5) by $g$, integrating over $D$, and adding the resulting equation, we obtain

$$
\begin{aligned}
\left(\frac{d \xi}{d t}, f\right)+\alpha_{1}^{-1}(t)((\xi \cdot \nabla) \xi, f)-\mu(\Delta \xi, f) \\
\quad+\alpha_{1}(t)(\nabla p, f)+\left(\frac{d \eta}{d t}, g\right) \\
\quad+\alpha_{1}^{-1}(t)((\xi \cdot \nabla) \eta, g)-k(\Delta \eta, g) \\
=\frac{\alpha_{1}(t)}{\alpha_{2}(t)}\left(e_{2} \eta, f\right) .
\end{aligned}
$$

Note that

$$
\begin{aligned}
\left(\frac{d \xi}{d t}, f\right)+\left(\frac{d \eta}{d t}, g\right) & =\left(\frac{d u}{d t}, y\right) \\
((\xi \cdot \nabla) \xi, f)+((\xi \cdot \nabla) \eta, g) & =-b(u, u, y) \\
-\mu(\Delta \xi, f)-k(\Delta \eta, g) & =a(u, y) .
\end{aligned}
$$

On the other hand,

$$
\begin{aligned}
\operatorname{div} f & =0, \\
f & =\left(f_{1}, f_{2}\right)^{T} .
\end{aligned}
$$


Then,

$$
(\nabla p, f)=0 .
$$

Therefore, (9) can also be written as

$$
\frac{d u}{d t}=-A u+\alpha_{1}^{-1}(t) B(u, u)+R(t) u .
$$

By the Galerkin method (see [14, Theorem 3.1]), we can prove that (21) has a unique solution $u(x, t)$, which satisfies the following:

(i) For all $t_{0} \in R$, if $u_{0} \in \mathscr{H}$, then

$$
u \in C\left(\left[t_{0}, \infty\right) ; \mathscr{H}\right) \cap L_{\text {Loc }}^{2}\left(\left[t_{0}, \infty\right) ; V\right) .
$$

(ii) Denoting such solution by $u\left(t, \omega ; t_{0}, u_{0}\right)$, for any $t \geq t_{0}$, the mapping

$$
u_{0} \longrightarrow u\left(t, \omega ; t_{0}, u_{0}\right)
$$

is continuous. Then, we define a stochastic flow $\varphi(t, \omega)$ by

$$
\begin{gathered}
\varphi(t, \omega) z_{0}=(\xi, \eta)^{T}=\left(\alpha_{1}(t) \mathscr{Q}_{\mathscr{H}_{1}} z\left(t, \omega ; t_{0}, z_{0}\right), \alpha_{2}(t)\right. \\
\left.\cdot \mathscr{Q}_{\mathscr{H}_{2}} z\left(t, \omega ; t_{0}, z_{0}\right)\right)^{T},
\end{gathered}
$$

where $z_{0}=z\left(t_{0}\right)=\left(v_{0}, T_{0}\right)^{T} \in \mathscr{H}$ and

$$
Q_{\mathscr{H}_{i}}: \mathscr{H}=\mathscr{H}_{1} \times \mathscr{H}_{2} \longrightarrow \mathscr{H}_{i}, \quad i=1,2
$$

are projection operators.

We can prove that $\varphi(t, \omega)$ is a continuous $\operatorname{RDS}$ determined by (1) easily.

\section{Random Attractor}

In this section, we will establish the existence of nonempty compact tempered random attractor for the RDS $\varphi(t, \omega)$ determined by (1). First we prove that $\varphi(t, \omega)$ possesses absorbing set in $\mathscr{H}$.

Lemma 1. Let $u=(\xi, \eta)^{T}$ be a solution of (4)-(7), for any $t_{0} \leq$ $t$, and one has

$$
\begin{aligned}
\|\xi\|^{2} \leq & \left\|\xi\left(t_{0}\right)\right\|^{2} e^{-\left(\mu / c_{0}^{2}\right)\left(t-t_{0}\right)} \\
& +\frac{c_{0}^{2}}{\mu} \int_{t_{0}}^{t} \frac{\alpha_{1}^{2}(s)}{\alpha_{2}^{2}(s)}\|\eta(s)\|^{2} e^{-\left(\mu / c_{0}^{2}\right)(t-s)} d s, \\
\|\eta\|^{2} \leq & \left\|\eta\left(t_{0}\right)\right\|^{2} e^{-\left(2 k / c_{1}^{2}\right)\left(t-t_{0}\right)} .
\end{aligned}
$$

Proof. Taking the scalar product of (4) with $\xi$ on $\mathscr{H}_{1}$

$$
\begin{gathered}
\left(\frac{d \xi}{d t}, \xi\right)+\alpha_{1}^{-1}(t)((\xi \cdot \nabla) \xi, \xi)-\mu(\Delta \xi, \xi) \\
+\alpha_{1}(t)(\nabla p, \xi)=\frac{\alpha_{1}(t)}{\alpha_{2}(t)}\left(e_{2} \eta, \xi\right) .
\end{gathered}
$$

By the incompressibility condition (6), we have

$$
\begin{aligned}
((\xi \cdot \nabla) \xi, \xi) & =0, \\
(\nabla p, \xi) & =0 .
\end{aligned}
$$

From (27), we obtain

$$
\frac{1}{2} \frac{d}{d t}\|\xi\|^{2}+\mu\|\nabla \xi\|^{2}=\frac{\alpha_{1}(t)}{\alpha_{2}(t)}\left(\eta, \xi_{2}\right) .
$$

Using Poincaré inequality, $\|\xi\| \leq c_{0}\|\nabla \xi\|$ ( $c_{0}$ is constant), we have

$$
\begin{aligned}
\frac{1}{2} \frac{d}{d t}\|\xi\|^{2}+\frac{\mu}{c_{0}^{2}}\|\xi\|^{2} & \leq \frac{\alpha_{1}(t)}{\alpha_{2}(t)}\|\eta\|\left\|\xi_{2}\right\| \leq \frac{\alpha_{1}(t)}{\alpha_{2}(t)}\|\eta\|\|\xi\| \\
& \leq \frac{c_{0}^{2} \alpha_{1}^{2}(t)}{2 \mu \alpha_{2}^{2}(t)}\|\eta\|^{2}+\frac{\mu}{2 c_{0}^{2}}\|\xi\|^{2} .
\end{aligned}
$$

Then,

$$
\frac{d}{d t}\|\xi\|^{2}+\frac{\mu}{c_{0}^{2}}\|\xi\|^{2} \leq \frac{c_{0}^{2} \alpha_{1}^{2}(t)}{\mu \alpha_{2}^{2}(t)}\|\eta\|^{2} .
$$

By the Gronwall inequality, for any $t_{0} \leq t$, we get that

$$
\begin{aligned}
\|\xi\|^{2} \leq & \left\|\xi\left(t_{0}\right)\right\|^{2} e^{-\left(\mu / c_{0}^{2}\right)\left(t-t_{0}\right)} \\
& +\frac{c_{0}^{2}}{\mu} \int_{t_{0}}^{t} \frac{\alpha_{1}^{2}(s)}{\alpha_{2}^{2}(s)}\|\eta(s)\|^{2} e^{-\left(\mu / c_{0}^{2}\right)(t-s)} d s .
\end{aligned}
$$

Taking the scalar product of (5) with $\eta$ on $\mathscr{H}_{2}$ as well

$$
\left(\frac{d \eta}{d t}, \eta\right)+\frac{1}{\alpha_{1}(t)}((\xi \cdot \nabla) \eta, \eta)-k(\Delta \eta, \eta)=0 .
$$

Since $((\xi \cdot \nabla) \eta, \eta)=0$, then

$$
\frac{d}{d t}\|\eta\|^{2}+2 k\|\nabla \eta\|^{2}=0 .
$$

Using Poincaré inequality $\|\eta\| \leq c_{1}\|\nabla \eta\|$, we have

$$
\frac{d}{d t}\|\eta\|^{2}+\frac{2 k}{c_{1}^{2}}\|\eta\|^{2} \leq 0
$$

By Gronwall inequality, for any $t_{0} \leq t$, we obtain that

$$
\|\eta\|^{2} \leq\left\|\eta\left(t_{0}\right)\right\|^{2} e^{-\left(2 k / c_{1}^{2}\right)\left(t-t_{0}\right)} .
$$

Lemma 2. There exist two positive random variables $r(\omega)$ and $C(\omega)$, such that, for all $\rho>0, t_{0} \leq t(\omega, \rho), t \in[-1,0]$, and $z_{0}=\left(v_{0}, T_{0}\right)^{T} \in \mathscr{H}$ with $\left\|z_{0}\right\|<\rho$, the solution $u=(\xi, \eta)^{T}$ of equations (4)-(7) satisfies

$$
\begin{gathered}
\left\|\eta\left(t, \omega ; t_{0}, \alpha_{2}\left(t_{0}\right) T_{0}\right)\right\|^{2} \leq C(\omega), \\
\left\|\xi\left(t, \omega ; t_{0}, \alpha_{1}\left(t_{0}\right) v_{0}\right)\right\|^{2} \leq r(\omega) .
\end{gathered}
$$


Proof. For $-1 \leq t \leq 0, t_{0} \leq-1$, from (26), we have

$$
\|\eta\|^{2} \leq \alpha_{2}^{2}(t)\left\|T\left(t_{0}\right)\right\|^{2} e^{\left(2 k / c_{1}^{2}\right)\left(1+t_{0}\right)}
$$

taking $t_{1}(\omega, \rho) \leq-1$, such that $\rho \alpha_{2}^{2}(t) e^{\left(2 k / c_{1}^{2}\right)\left(1+t_{1}\right)} \leq C(\omega)$.

Then, for any $t_{0} \leq t_{1}(\omega, \rho)$,

$$
\left\|\eta\left(t, \omega ; t_{0}, \alpha_{2}\left(t_{0}\right) T_{0}\right)\right\|^{2} \leq C(\omega) .
$$

From (26), we find

$$
\begin{aligned}
\|\xi\|^{2} \leq & \left\|\xi\left(t_{0}\right)\right\|^{2} e^{\left(\mu / c_{0}^{2}\right)\left(1+t_{0}\right)} \\
& +\frac{c_{0}^{2}}{\mu} \int_{t_{0}}^{t} \frac{\alpha_{1}^{2}(s)}{\alpha_{2}^{2}(s)}\|\eta(s)\|^{2} e^{\left(\mu / c_{0}^{2}\right)(1+s)} d s, \\
\lim _{s \rightarrow-\infty} \frac{W_{1}(s)}{s}= & 0, \quad P \text {-a.s. }
\end{aligned}
$$

Then, $\alpha_{1}^{2}\left(t_{0}\right) e^{\mu t_{0} / c_{0}^{2}} \rightarrow 0$, as $t_{0} \rightarrow-\infty$.

Thus, there exists $t_{2}(\omega, \rho) \leq-1$, for $t_{0} \leq t_{2}$ :

$$
\left\|v_{0}\right\| \alpha_{1}^{2}\left(t_{0}\right) e^{\mu t_{0} / c_{0}^{2}} \leq \rho^{2} \alpha_{1}^{2}\left(t_{0}\right) e^{\mu t_{0} / c_{0}^{2}} \leq 1 .
$$

By (37), we infer that $\forall t_{0} \leq t_{1}, t \in[-1,0], P$-a.s is

$$
\begin{aligned}
& \int_{t_{0}}^{t} \frac{\alpha_{1}^{2}(s)}{\alpha_{2}^{2}(s)}\|\eta(s)\|^{2} e^{\left(\mu / c_{0}^{2}\right)(1+s)} d s \\
& \leq C^{2}(\omega) \int_{-\infty}^{0} \frac{\alpha_{1}^{2}(s)}{\alpha_{2}^{2}(s)} e^{\left(\mu / c_{0}^{2}\right)(1+s)} d s .
\end{aligned}
$$

Since

$$
\frac{\alpha_{1}^{2}(s)}{\alpha_{2}^{2}(s)}=e^{2 b_{2} W_{2}(s)-2 b_{1} W_{1}(s)}=e^{b_{3} W(s)}
$$

then $\left(\alpha_{1}^{2}(s) / \alpha_{2}^{2}(s)\right) e^{\mu s / c_{0}^{2}} \rightarrow 0, s \rightarrow-\infty$. Therefor,

$$
\int_{t_{0}}^{t} \frac{\alpha_{1}^{2}(s)}{\alpha_{2}^{2}(s)}\|\eta(s)\|^{2} e^{\left(\mu / c_{0}^{2}\right)(1+s)} d s<\infty .
$$

Put

$$
r(\omega)=e^{\mu / c_{0}^{2}}\left[1+\frac{c_{0}^{2}}{\mu} \int_{t_{0}}^{t} \frac{\alpha_{1}^{2}(s)}{\alpha_{2}^{2}(s)}\|\eta(s)\|^{2} e^{\mu s / c_{0}^{2}} d s\right]
$$

Then, $r(\omega)$ exists and it is tempered.

Getting $t(\omega, \rho)=\min \left\{t_{1}, t_{2}\right\}$, for any $t \geq t_{0}$,

$$
\begin{gathered}
\left\|\eta\left(t, \omega ; t_{0}, \alpha_{2}\left(t_{0}\right) T_{0}\right)\right\|^{2} \leq C(\omega), \\
\left\|\xi\left(t, \omega ; t_{0}, \alpha_{1}\left(t_{0}\right) v_{0}\right)\right\|^{2} \leq r(\omega) .
\end{gathered}
$$

Now we are going to prove that $\varphi(t, \omega)$ is asymptotically compact.
Lemma 3. There exist two positive random variables $C_{1}(\omega)$ and $r_{1}(\omega)$, such that, for all $\rho>0, t_{0} \leq t(\omega, \rho), t \in[-1,0]$, and $z_{0}=\left(v_{0}, T_{0}\right)^{T} \in \mathscr{H}$ with $\left\|z_{0}\right\|<\rho$, the solution $u=(\xi, \eta)^{T}$ of equations (4)-(7) satisfies

$$
\begin{aligned}
& \int_{-1}^{0}\left\|\nabla \eta\left(s, \omega ; t_{0}, \alpha_{2}(s) T_{0}\right)\right\|^{2} d s \leq C_{1}(\omega), \\
& \int_{-1}^{0}\left\|\nabla \xi\left(s, \omega ; t_{0}, \alpha_{1}(s) v_{0}\right)\right\|^{2} d s \leq r_{1}(\omega) .
\end{aligned}
$$

Proof. Integrating (34) on $[-1,0]$, we have

$$
\|\eta(0)\|^{2}-\|\eta(-1)\|^{2}+2 k \int_{-1}^{0}\|\nabla \eta\|^{2} d s=0 .
$$

From Lemma 1, we know that

$$
\begin{aligned}
\int_{-1}^{0}\|\nabla \eta\|^{2} d s & \leq \frac{1}{2 k}\left(\|\eta(-1)\|^{2}-\|\eta(0)\|^{2}\right) \leq \frac{C(\omega)}{k} \\
& =C_{1}(\omega) .
\end{aligned}
$$

We get from (29),

$$
\frac{1}{2} \frac{d}{d t}\|\xi\|^{2}+\mu\|\nabla \xi\|^{2} \leq \frac{c_{0}^{2} \alpha_{1}^{2}(t)}{2 \mu \alpha_{2}^{2}(t)}\|\eta\|^{2}+\frac{\mu}{2}\|\nabla \xi\|^{2}
$$

then

$$
\frac{d}{d t}\|\xi\|^{2}+\mu\|\nabla \xi\|^{2} \leq \frac{c_{0}^{2} \alpha_{1}^{2}(t)}{\mu \alpha_{2}^{2}(t)}\|\eta\|^{2}
$$

Integrating the above formula over $[-1,0]$,

$$
\begin{gathered}
\|\xi(0)\|^{2}-\|\xi(-1)\|^{2}+\mu \int_{-1}^{0}\|\nabla \xi(s)\|^{2} d s \\
\leq \int_{-1}^{0} \frac{c_{0}^{2} \alpha_{1}^{2}(s)}{\mu \alpha_{2}^{2}(s)}\|\eta(s)\|^{2} d s
\end{gathered}
$$

then

$$
\begin{aligned}
& \int_{-1}^{0}\|\nabla \xi(s)\| d s \leq \frac{1}{\mu}\left(\|\xi(-1)\|^{2}-\|\xi(0)\|^{2}\right. \\
& \left.+\int_{-1}^{0} \frac{c_{0}^{2} \alpha_{1}^{2}(s)}{\mu \alpha_{2}^{2}(s)}\|\eta(s)\|^{2} d s\right) .
\end{aligned}
$$

Let

$$
r_{1}(\omega)=\frac{2 r(\omega)}{\mu}+\frac{c_{0}^{2}}{\mu^{2}} \int_{-1}^{0} \frac{\alpha_{1}^{2}(s)}{\alpha_{2}^{2}(s)}\|\eta(s)\|^{2} d s
$$

then

$$
\int_{-1}^{0}\left\|\nabla \xi\left(s, \omega ; t_{0}, \alpha_{1}(s) v_{0}\right)\right\|^{2} d s \leq r_{1}(\omega) .
$$


Lemma 4. There exist two positive random variables $C_{2}(\omega)$ and $r_{2}(\omega)$, such that, for all $\rho>0, t_{0} \leq t(\omega, \rho), t \in[-1,0]$, and $z_{0}=\left(v_{0}, T_{0}\right)^{T} \in \mathscr{H}$ with $\left\|z_{0}\right\|<\rho$, the solution $u=(\xi, \eta)^{T}$ of (4)-(7) satisfies

$$
\begin{gathered}
\left\|\nabla \eta\left(0, \omega ; t_{0}, \alpha_{2}(s) T_{0}\right)\right\|^{2} \leq C_{2}(\omega), \\
\left\|\nabla \xi\left(0, \omega ; t_{0}, \alpha_{1}(s) v_{0}\right)\right\|^{2} \leq r_{2}(\omega) .
\end{gathered}
$$

Proof. Taking the scalar product of (5) with $-\Delta \eta$ on $\mathscr{H}_{2}$, then

$$
\begin{aligned}
& \frac{1}{2} \frac{d}{d t}\|\nabla \eta\|^{2}+k\|\nabla \eta\|^{2} \leq \alpha_{1}^{-1}(t)|((\xi \cdot \nabla) \eta,-\Delta \eta)| \\
& \quad \leq \alpha_{1}^{-1}(t)\|(\xi \cdot \nabla) \eta\|\|\Delta \eta\| .
\end{aligned}
$$

Since

$$
\begin{aligned}
\|(\xi \cdot \nabla) \eta\| & \leq\|\xi\|_{L^{4}}\|\nabla \eta\|_{L^{4}} \\
& \leq c_{2}\|\nabla \xi\|^{1 / 2}\|\xi\|^{1 / 2}\|\nabla \eta\|^{1 / 2}\|\Delta \eta\|^{1 / 2},
\end{aligned}
$$

then we have

$$
\begin{aligned}
& \frac{1}{2} \frac{d}{d t}\|\nabla \eta\|^{2}+k\|\Delta \eta\|^{2} \\
& \quad \leq \alpha_{1}^{-1}(t) c_{2}\|\nabla \xi\|^{1 / 2}\|\xi\|^{1 / 2}\|\nabla \eta\|^{1 / 2}\|\Delta \eta\|^{3 / 2} \\
& \quad \leq \frac{27 c_{2}^{4}}{256 k^{3} \alpha_{1}^{4}(t)}\|\xi\|^{2}\|\nabla \xi\|^{2}\|\nabla \eta\|^{2}+k\|\Delta \eta\|^{2} .
\end{aligned}
$$

Hence,

$$
\frac{d}{d t}\|\nabla \eta\|^{2} \leq \frac{27 c_{2}^{4}}{128 k^{3} \alpha_{1}^{4}(t)}\|\xi\|^{2}\|\nabla \xi\|^{2}\|\nabla \eta\|^{2} .
$$

Using the Gronwall inequality over an arbitrary interval $[s, 0] \subset[-1,0]$, we obtain

$$
\|\nabla \eta(0)\|^{2} \leq\|\nabla \eta(s)\|^{2} e^{\int_{s}^{0} h(\tau) d \tau} .
$$

Here, $h(\tau)=\left(27 c_{2}^{4} / 128 k^{3} \alpha_{1}^{4}(\tau)\right)\|\xi(\tau)\|^{2}\|\nabla \xi(\tau)\|^{2}$.

Integrating the above formula over the interval $[-1,0]$ for $s$, we obtain

$$
\|\nabla \eta(0)\|^{2} \leq e^{\int_{-1}^{0} h(\tau) d \tau} \int_{-1}^{0}\|\nabla \eta(s)\|^{2} d s .
$$

Let

$$
C_{3}(\omega)=e^{\int_{-1}^{0} h(\tau) d \tau} C_{1}(\omega) .
$$

Thus, $C_{2}(\omega)$ exists and it is tempered and we have

$$
\left\|\nabla \eta\left(0, \omega ; t_{0}, \alpha_{2}\left(t_{0}\right) T_{0}\right)\right\|^{2} \leq C_{2}(\omega) .
$$

Taking the scalar product of (4) with $-\Delta \xi$ on $\mathscr{H}_{1}$

$$
\begin{gathered}
\left(\frac{d \xi}{d t},-\Delta \xi\right)+\alpha_{1}(t)^{-1}((\xi \cdot \nabla) \xi,-\Delta \xi)-\mu(\Delta \xi,-\Delta \xi) \\
+\alpha_{1}(t)(\nabla p,-\Delta \xi)=\left(\frac{\alpha_{1}(t)}{\alpha_{2}(t)} e_{2} \eta,-\Delta \xi\right) .
\end{gathered}
$$

Since $(\nabla p,-\Delta \xi)=0$, then

$$
\begin{aligned}
\frac{1}{2} \frac{d}{d t} & \|\nabla \xi\|^{2}+\mu\|\Delta \xi\|^{2} \\
\leq & \alpha_{1}^{-1}(t)((\xi \cdot \nabla) \xi, \Delta \xi)+\frac{\alpha_{1}(t)}{\alpha_{2}(t)}\left(e_{2} \eta, \Delta \xi\right) \\
\leq & \frac{c_{3}}{\alpha_{1}(t)}\|\xi\|^{1 / 2}\|\nabla \xi\|\|\Delta \xi\|^{3 / 2}+\frac{\alpha_{1}(t)}{\alpha_{2}(t)}\|\eta\|\|\Delta \xi\| \\
\leq & \frac{27 c_{3}^{4}}{32 \alpha_{1}^{4}(t) \mu^{3}}\|\xi\|^{2}\|\nabla \xi\|^{4}+\frac{\alpha_{1}^{2}(t)}{2 \mu \alpha_{2}^{2}(t)}\|\eta\|^{2} \\
& +\mu\|\Delta \xi\|^{2} .
\end{aligned}
$$

Namely,

$$
\begin{aligned}
\frac{d}{d t}\|\nabla \xi\|^{2} & \leq \frac{27 c_{3}^{4}}{16 \alpha_{1}^{4}(t) \mu^{3}}\|\xi\|^{2}\|\nabla \xi\|^{4}+\frac{\alpha_{1}^{2}(t)}{\mu \alpha_{2}^{2}(t)}\|\eta\|^{2} \\
& \leq G_{1}(t)\|\nabla \xi\|^{2}+G_{2}(t),
\end{aligned}
$$

where $G_{1}(t)=\left(27 c_{3}^{4} / 16 \alpha_{1}^{4}(t) \mu^{3}\right)\|\xi\|^{2}\|\nabla \xi\|^{2}$ and $G_{2}(t)=$ $\left(\alpha_{1}^{2}(t) / \mu \alpha_{2}^{2}(t)\right)\|\eta\|^{2}$.

By the Gronwall inequality over an arbitrary interval $[s, 0] \subset[-1,0]$, we obtain

$$
\begin{aligned}
\|\nabla \xi(0)\|^{2} \leq & e^{\int_{s}^{0} G_{1}(\sigma) d \sigma}\|\nabla \xi(s)\|^{2} \\
& \quad \int_{s}^{0} G_{2}(\tau) e^{\int_{\tau}^{0} G_{1}(\sigma) d \sigma} d \tau .
\end{aligned}
$$

Integrating the above formula over the interval $[-1,0]$ for $s$, we have

$$
\begin{aligned}
\|\nabla \xi(0)\|^{2} \leq & e^{\int_{-1}^{0} G_{1}(\sigma) d \sigma} \int_{-1}^{0}\|\nabla \xi(s)\|^{2} d s \\
& +\int_{-1}^{0} G_{2}(\tau) e^{\int_{-1}^{0} G_{1}(\sigma) d \sigma} d \tau .
\end{aligned}
$$

Since

$$
\begin{aligned}
\int_{-1}^{0} G_{1}(\sigma) d \sigma & \leq \frac{27 c_{3}^{4}}{16 \alpha_{1}^{4}(t) \mu^{3}} r(\omega) \int_{-1}^{0}\|\nabla \xi(\sigma)\|^{2} d \sigma \\
& \leq \frac{27 c_{3}^{4}}{16 \alpha_{1}^{4}(t) \mu^{3}} r(\omega) r_{1}(\omega),
\end{aligned}
$$

let

$$
\begin{aligned}
& r_{2}(\omega) \\
& \quad=e^{\left(27 c_{3}^{4} / 16 \alpha_{1}^{4}(t) \mu^{3}\right) r(\omega) r_{1}(\omega)}\left[r_{1}(\omega)+\int_{-1}^{0} G_{2}(\tau) d \tau\right] .
\end{aligned}
$$

Thus, $r_{2}(\omega)$ exists and it is tempered. Then,

$$
\left\|\nabla \xi\left(0, \omega ; t_{0}, \alpha_{1}\left(t_{0}\right) u_{0}\right)\right\|^{2} \leq r_{2}(\omega) .
$$

Let $\mathscr{B}(\omega)$ be a random closed ball centered at 0 with radius $\sqrt{C_{2}(\omega)+r_{2}(\omega)}$.

Then, $\mathscr{B}(\omega)$ is a random absorbing set for $\varphi(t, \omega)$ in $V$. Since $V$ embedding $\mathscr{H}$ is compact, then $\operatorname{RDS} \varphi(t, \omega)$ is asymptotically compact in $\mathscr{H}$. Thus, the RDS determined by (1) possesses a random attractor. 


\section{Hausdorff Dimensions of Random Attractors}

In this section, we will estimate the Hausdorff dimensions of $\mathscr{A}(\omega)$. Now, we assume that $u_{0}, u_{1}(t), u_{2}(t), \chi$ are $\mathscr{F}_{t}$ measurable and prove that the $\operatorname{RDS} \varphi(t, \omega)$ is differentiable on $\mathscr{A}(\omega)$.

Theorem 5. The RDS $\varphi(t, \omega)$ determined by (21) is almost surely uniformly differentiable on $\mathscr{A}(\omega), \omega \in \Omega$, and its derivative is $D \varphi\left(t, t_{0}, \omega ; u_{0}\right) \chi=U(t), t_{0} \leq t$, which is the solution of the following variational equation:

$$
\begin{aligned}
\frac{d U}{d t} & =-A U+F(t, u) U, \\
U\left(t_{0}\right) & =\chi,
\end{aligned}
$$

where $F(t, u) U=\alpha_{1}^{-1}(t)[B(u, U)+B(U, u)]+R(t) U, u=$ $\varphi\left(t, t_{0} ; \omega\right) u_{0}, t_{0} \leq t$.

Proof. Let $u_{1}(t)=\varphi\left(t, t_{0} ; \omega\right) u_{0}^{(1)}, u_{2}(t)=\varphi\left(t, t_{0} ; \omega\right) u_{0}^{(2)}$, and $\delta(t)=u_{1}(t)-u_{2}(t)$, and we have

$$
\begin{aligned}
\frac{d}{d t} \delta(t)= & -A \delta(t) \\
& +\alpha_{1}^{-1}(t)\left[B\left(u_{1}, \delta(t)\right)+B\left(\delta(t), u_{2}\right)\right] \\
& +R(t) \delta(t) \\
\frac{d U}{d t}= & -A(U)+\alpha_{1}^{-1}(t)\left[B\left(u_{1}, U\right)+B\left(U, u_{2}\right)\right] \\
& +R(t) U .
\end{aligned}
$$

We also set $l(t)=\delta(t)-U(t)$, and then

$$
\begin{aligned}
& \frac{d l(t)}{d t}=-A l(t)+\alpha_{1}^{-1}(t) \\
& \cdot\left[B\left(u_{2}, l(t)\right)+B\left(l(t), u_{2}\right)+B(\delta(t), \delta(t))\right] \\
& \quad+R(t) l(t) .
\end{aligned}
$$

Taking the scalar product of (75) with $l(t)$ on $\mathscr{H}$,

$$
\begin{aligned}
& \frac{1}{2} \frac{d}{d t}\|l(t)\|^{2} \leq-\|\nabla l(t)\|^{2}+\alpha_{1}^{-1}(t) \\
& \cdot\left[\left(B\left(l(t), u_{2}\right), l(t)\right)+(B(\delta(t), \delta(t)), l(t))\right] \\
& \quad+R(t)\|l(t)\|^{2} \\
& \quad \leq \frac{c_{4}}{\alpha_{1}(t)}\left(\left\|\nabla u_{2}\right\|\|l(t)\|^{2}+\|\delta(t)\|\|\nabla \delta(t)\|\|l(t)\|\right) \\
& +R(t)\|l(t)\|^{2} \leq K(t)\|l(t)\|^{2}+c_{5}\|\delta(t)\|^{2} \\
& \cdot\|\nabla \delta(t)\|^{2} .
\end{aligned}
$$

Here, $K(t)$ is the function about $t$. By the Gronwall inequality, we obtain

$$
\begin{aligned}
\|l(t)\|^{2} & \leq \int_{t_{0}}^{t} e^{\int_{s}^{t} 2 K(s)(\tau) d \tau}\left(2 c_{5}\|\delta(s)\|^{2}\|\nabla \delta(s)\|^{2}\right) d s \\
& \leq K_{1}(t) \int_{t_{0}}^{t}\|\delta(s)\|^{2}\|\nabla \delta(s)\|^{2} d s .
\end{aligned}
$$

Taking the scalar product of (74) with $\delta(t)$ on $\mathscr{H}$,

$$
\begin{aligned}
\frac{1}{2} \frac{d}{d t}\|\delta(t)\|^{2} \leq & -\|\nabla \delta(t)\|^{2} \\
& +\alpha_{1}^{-1}(t)\left(B\left(\delta(t), u_{2}\right), \delta(t)\right) \\
& +R(t)\|\delta(t)\|^{2} \\
\leq & -\|\nabla \delta(t)\|^{2} \\
& +\frac{c_{6}}{\alpha_{1}(t)}\|\delta(t)\|\left\|\nabla u_{2}\right\|\|\nabla \delta(t)\| \\
& +R(t)\|\delta(t)\|^{2} \\
\leq & -\|\nabla \delta(t)\|^{2}+\frac{1}{2}\|\nabla \delta(t)\|^{2} \\
& +\frac{c_{6}}{2 \alpha_{1}^{2}(t)}\|\delta(t)\|^{2}\left\|\nabla u_{2}\right\|^{2} \\
& +R(t)\|\delta(t)\|^{2} \\
\leq & -\frac{1}{2}\|\nabla \delta(t)\|^{2}+K_{2}(t)\|\delta(t)\|^{2} .
\end{aligned}
$$

Then,

$$
\frac{d}{d t}\|\delta(t)\|^{2} \leq 2 K_{2}(t)\|\delta(t)\|^{2} .
$$

By the Gronwall inequality, we get

$$
\begin{aligned}
\|\delta(t)\|^{2} & \leq e^{\int_{t_{0}}^{t} 2 K_{2}(s) d s}\left\|u_{0}^{(1)}-u_{0}^{(2)}\right\|^{2} \\
& \leq K_{3}(t)\left\|u_{0}^{(1)}-u_{0}^{(2)}\right\|^{2} .
\end{aligned}
$$

We get from (80) that

$$
\begin{aligned}
\int_{t_{0}}^{t}\|\nabla \delta(t)\|^{2} d s \leq & \left\|u_{0}^{(1)}-u_{0}^{(2)}\right\|^{2} \\
& +\int_{t_{0}}^{t} 2 K_{2}(s)\|\delta(s)\|^{2} d s \\
\leq & \left\|u_{0}^{(1)}-u_{0}^{(2)}\right\|^{2} \\
& +\left\|u_{0}^{(1)}-u_{0}^{(2)}\right\|^{2} \int_{t_{0}}^{t} 2 K_{2}(s) K_{3}(s) d s \\
\leq & K_{4}(t)\left\|u_{0}^{(1)}-u_{0}^{(2)}\right\|^{2} .
\end{aligned}
$$


From (80) to (81), we have

$$
\|l(t)\|^{2} \leq K_{5}(t)\left\|u_{0}^{(1)}-u_{0}^{(2)}\right\|^{4} .
$$

The proof is completed.

Theorem 6. Set

$$
d=\min \left\{d:-\sum_{j=1}^{d} \lambda_{j}+c_{9} d<0, c_{9} \in R^{+}\right\} .
$$

Then the Hausdorff dimension of random attractor $\mathscr{A}(t, \omega)$ satisfies

$$
\operatorname{dim}_{H}(\mathscr{A}(t, \omega)) \leq d, \quad \forall t \in R .
$$

Proof. Taking the scalar product of (74) with $U(t)$ on $\mathscr{H}$,

$$
\begin{aligned}
\frac{1}{2} \frac{d}{d t}\|U\|^{2}= & -\|\nabla U\|^{2}+\alpha_{1}^{-1}(t)(B(U, u), U) \\
& +R(t)\|U\|^{2} \\
\leq & \alpha_{1}^{-1}(t)(B(U, u), U)+R(t)\|U\|^{2} \\
\leq & \frac{c_{7}}{\alpha_{1}(t)}\|U\|^{2}\|\nabla u\|+R(t)\|U\|^{2} .
\end{aligned}
$$

Then,

$$
\frac{d}{d t}\|U\|^{2} \leq 2 K_{6}(t)\|U\|^{2} .
$$

By the Gronwall inequality, we get

$$
\|U(t)\|^{2} \leq e^{\int_{t_{0}}^{t} 2 K_{6}(s) d s}\left\|U\left(t_{0}\right)\right\|^{2}
$$

which implies that

$$
\left\|D \varphi\left(t, t_{0}, u_{0} ; \omega\right)\right\| \leq e^{\int_{t_{0}}^{t} 2 K_{6}(s) d s} .
$$

Since

$$
a_{1}\left(D \varphi\left(t, t_{0}, u_{0} ; \omega\right)\right)=\left\|D \varphi\left(t, t_{0}, u_{0} ; \omega\right)\right\|,
$$

then

$$
a_{1}\left(D \varphi\left(t, t_{0}, u_{0} ; \omega\right)\right) \leq e^{\int_{t_{0}}^{t} 2 K_{6}(s) d s} .
$$

Taking $a^{\prime}(\omega)=\max \left\{1, e^{\int_{t_{0}}^{t} 2 K_{6}(s) d s}\right\}$, then we have

$$
\begin{gathered}
a_{1}(\omega)\left(D \varphi\left(t, 0, u_{0} ; \omega\right)\right) \leq a_{1}^{\prime}(\omega), \\
E\left(\ln a_{1}^{\prime}(\omega)\right)<\infty .
\end{gathered}
$$

Assume that $U_{j}(t)=D \varphi\left(t, t_{0}, u_{0} ; \omega\right) \chi_{j}$, here $\chi_{j} \in \mathscr{H}$, and $i=1,2, \ldots, d . Q_{d}(t)$ is the orthogonal projection in $\mathscr{H}$ onto the space spanned by $U_{1}(t), U_{2}(t), \ldots, U_{d}(t)$. Let $\left\{\Psi_{j}\right\}, j=$ $1,2, \ldots, d$, be an orthonormal basis in $Q_{d}(t) \mathscr{H}$. Then,

$$
\operatorname{Tr}\{-A+F(t, u)\} \circ Q_{d}(t)
$$

$$
\begin{aligned}
& =\sum_{j=1}^{d}\left(\{-A+F(t, u)\} \Psi_{j}, \Psi_{j}\right) \leq \sum_{j=1}^{d}\left\{\left(-A \Psi_{j}, \Psi_{j}\right)\right. \\
& \left.+\alpha_{1}^{-1}(t)\left(B\left(\Psi_{j}, u(t)\right), \Psi_{j}\right)+R(t)\left(\Psi_{j}, \Psi_{j}\right)\right\} \\
& \leq-\sum_{j=1}^{d} \lambda_{j}\left\|\Psi_{j}\right\|^{2}+\sum_{j=1}^{d}\left(c_{8} e^{b_{1} W_{1}(t)}\left\|\Psi_{j}\right\|^{2}\right. \\
& \left.+e^{b_{3} W_{3}(t)}\left\|\Psi_{j}\right\|^{2}\right) \leq-\sum_{j=1}^{d} \lambda_{j}+\sum_{j=1}^{d}\left(c_{8} e^{b_{1} W_{1}(t)}\right. \\
& \left.+e^{b_{3} W_{3}(t)}\right) .
\end{aligned}
$$

Set

$$
\begin{aligned}
& {\omega_{d}}\left(D \varphi\left(t, 0, u_{0} ; \omega\right)\right) \\
& \quad \leq \exp \left\{-\sum_{j=1}^{d} \lambda_{j}+\sum_{j=1}^{d}\left(c_{8} e^{b_{1} W_{1}(t)}+e^{b_{3} W_{3}(t)}\right)\right\} .
\end{aligned}
$$

Then,

$$
\begin{aligned}
E[ & \left.\ln \left(\sup _{u_{0} \in \mathscr{A}(\omega)} \omega\left(D \varphi\left(t, t_{0}, u_{0} ; \omega\right)\right)\right)\right] \\
\leq & -\sum_{j=1}^{d} \lambda_{j} \\
& +\int_{0}^{1}\left\{\sum_{j=1}^{d}\left(c_{8} E\left[e^{b_{1} W_{1}(t)}\right]+E\left[e^{b_{3} W_{3}(t)}\right]\right)\right\} d t \\
\leq & -\sum_{j=1}^{d} \lambda_{j}+c_{9} d .
\end{aligned}
$$

We can get that

$$
E\left[\ln \left(\sup _{u_{0} \in \mathscr{A}(\omega)} \Phi\left(D \varphi\left(t, t_{0}, u_{0} ; \omega\right)\right)\right)\right]<0
$$

when

$$
-\sum_{j=1}^{d} \lambda_{j}+c_{9} d<0, \quad c_{9} \in R^{+} .
$$

In this case, the Hausdorff dimension of random attractor $\mathscr{A}(t, \omega)$ satisfies

$$
\operatorname{dim}_{H}(\mathscr{A}(t, \omega)) \leq d, \quad \forall t \in R .
$$




\section{Competing Interests}

The authors declare that they have no competing interests.

\section{Acknowledgments}

This work is partially supported by the National Natural Science Foundation of China (Grant no. 11501373) and the Natural Science Foundation of Guangdong Province (no. 2016A030310019 and no. 2016A03030742), Guangdong Provincial culture of seedling of China (no. 2013LYM0081), the Education Research Platform Project of Guangdong Province (no. 2014KQNCX208), the Education Reform Project of Guangdong Province (no. 2015558), the Shaoguan Science and Technology Foundation (no. 20157201 and no. 20167201), and Education Reform Project of Shaoguan University (no. SYJY20121361 and no. SYJY20141576).

\section{References}

[1] X. Pu and B. Guo, "Global well-posedness of the stochastic 2D Boussinesq equations with partial viscosity," Acta Mathematica Scientia. Series B. English Edition, vol. 31, no. 5, pp. 1968-1984, 2011.

[2] Y. R. Li and B. L. Guo, "Random attractors of Boussinesq equations with multiplicative noise," Acta Mathematica Sinica, English Series, vol. 25, no. 3, pp. 481-490, 2009.

[3] W. Q. Zhao and Y. R. Li, "Asymptotic behavior of solutions to stochastic Boussinesq equations with additive white noises," Acta Mathematica Sinica. Chinese Series, vol. 56, no. 1, pp. 1-14, 2013.

[4] H. Crauel and F. Flandoli, "Hausdorff dimension of invariant sets for random dynamical systems," Journal of Dynamics and Differential Equations, vol. 10, no. 3, pp. 449-474, 1998.

[5] A. Debussche, "Hausdorff dimension of a random invariant set," Journal de Mathématiques Pures et Appliquées, vol. 77, no. 10, pp. 967-988, 1998.

[6] X. Fan and H. Chen, "Attractors for the stochastic reactiondiffusion equation driven by linear multiplicative noise with a variable coefficient," Journal of Mathematical Analysis and Applications, vol. 398, no. 2, pp. 715-728, 2013.

[7] X. Fan, "Random attractor for a damped sine-Gordon equation with white noise," Pacific Journal of Mathematics, vol. 216, no. 1, pp. 63-76, 2004.

[8] C. Sun and H. Gao, "Hausdorff dimension of random attractor for stochastic Navier-Stokes-Voight equations and primitive equations," Dynamics of Partial Differential Equations, vol. 7, no. 4, pp. 307-326, 2010.

[9] S. Gala, "On the regularity criterion of strong solutions to the 3D Boussinesq equations," Applicable Analysis, vol. 90, no. 12, pp. 1829-1835, 2011.

[10] S. Gala and M. A. Ragusa, "Logarithmically improved regularity criterion for the Boussinesq equations in BESov spaces with negative indices," Applicable Analysis, vol. 95, no. 6, pp. 12711279, 2016.

[11] S. Gala, Z. Guo, and M. A. Ragusa, "A remark on the regularity criterion of Boussinesq equations with zero heat conductivity," Applied Mathematics Letters, vol. 27, pp. 70-73, 2014.

[12] S. Gala and Z. Zhang, "Osgood type regularity criterion for the 3D Newton-Boussinesq equation," Electronic Journal of Differential Equations, vol. 2013, no. 223, 6 pages, 2013.
[13] J. Fan and Y. Zhou, "A note on regularity criterion for the 3D Boussinesq system with partial viscosity", Applied Mathematics Letters, vol. 22, no. 5, pp. 802-805, 2009.

[14] R. Temam, Infinite-dimensional Dynamical Systems in Mechanics And Physics, vol. 68 of Applied Mathematical Sciences, Springer, New York, NY, USA, 1988. 


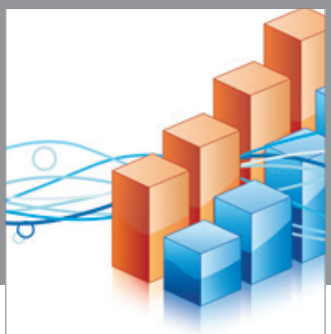

Advances in

Operations Research

vatem alat4

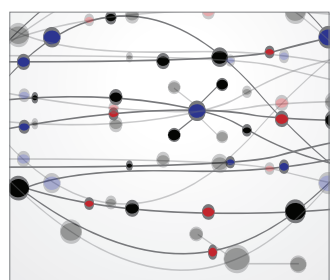

\section{The Scientific} World Journal
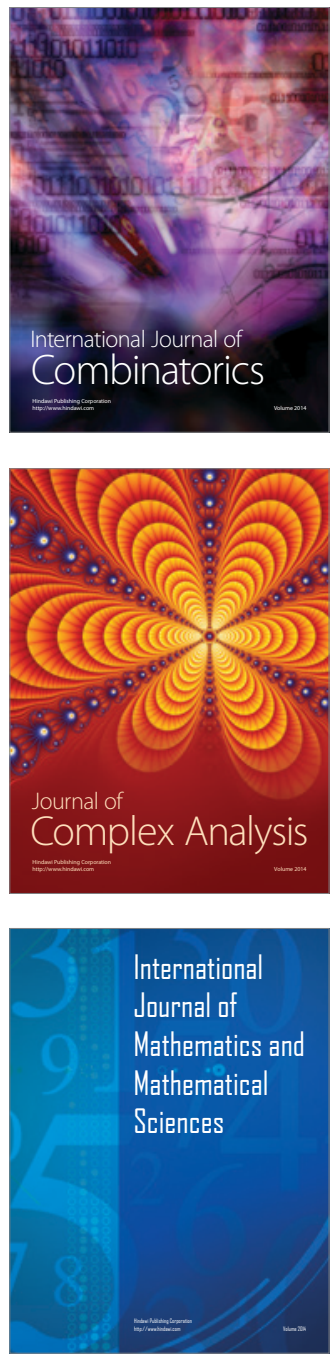
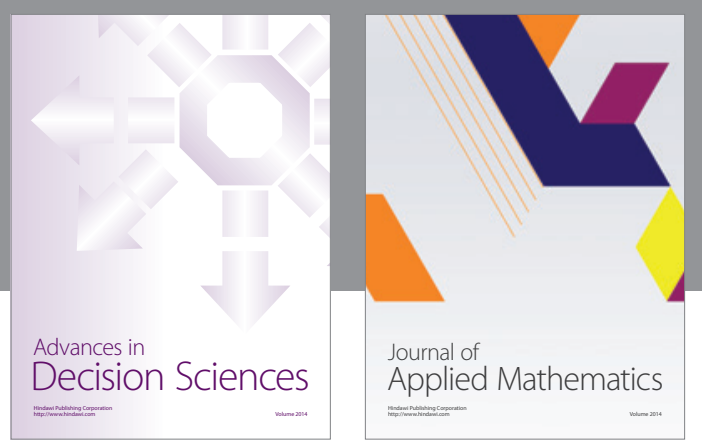

Algebra

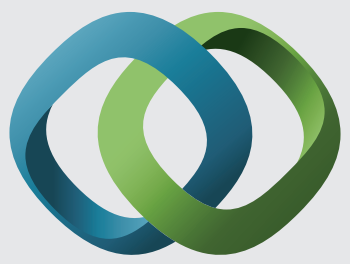

\section{Hindawi}

Submit your manuscripts at

http://www.hindawi.com
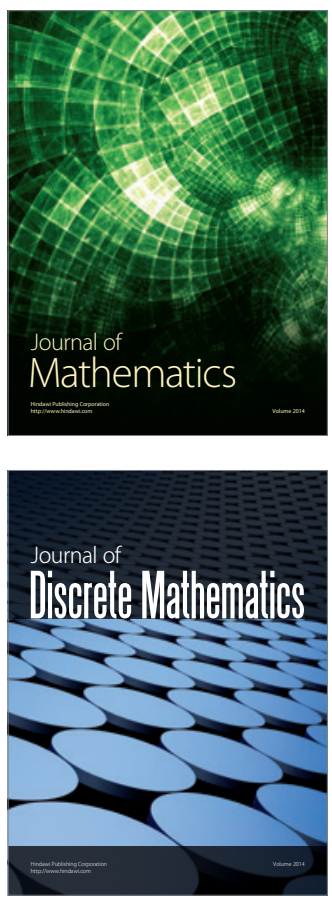

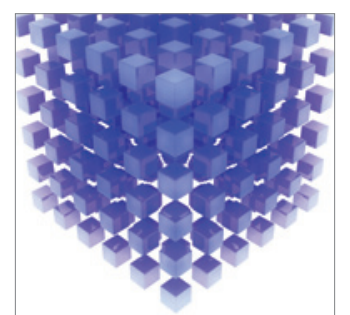

Mathematical Problems in Engineering
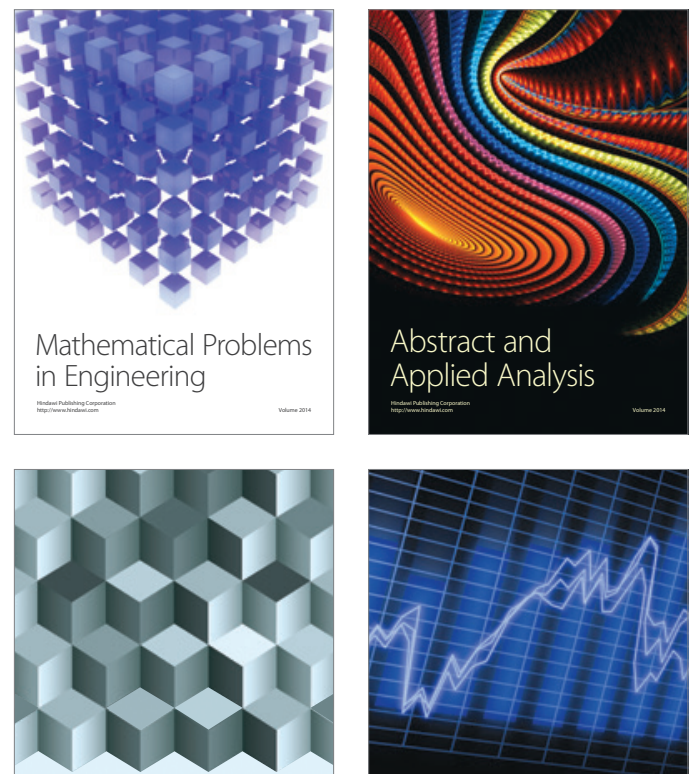

Journal of

Function Spaces

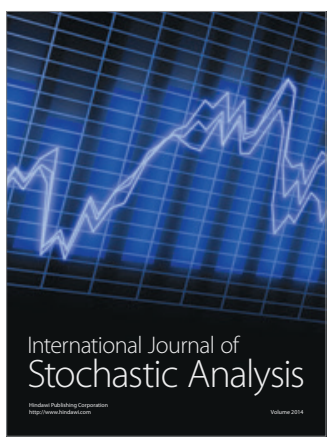

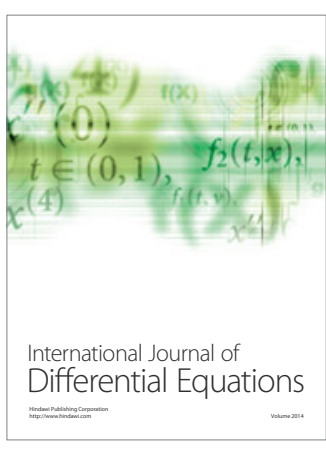
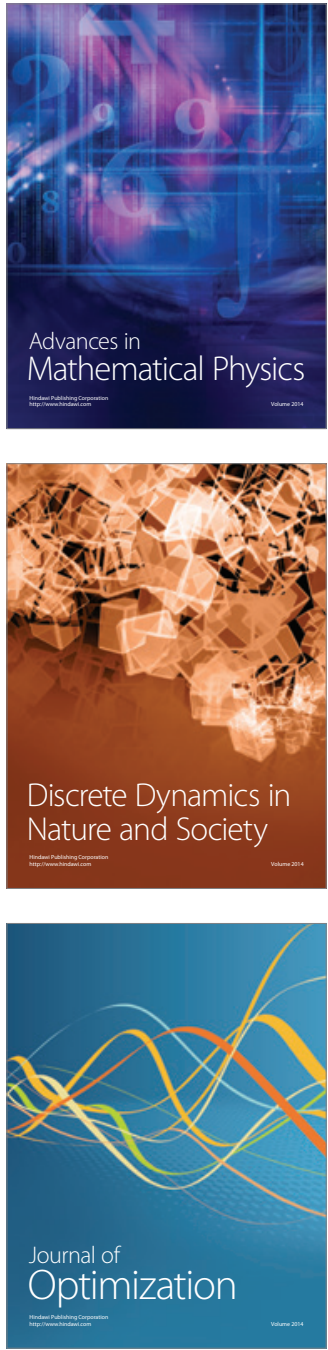